\title{
Deterioração da madeira de Eucalyptus e Corymbia em ensaio de campo
}

\author{
Manolo Trindade Quintilhan*, Weslley Candido de Oliveira, Aylson Costa Oliveira, Bárbara Luísa \\ Corradi Pereira, Romulo Môra, Adrianna Amorim de Sousa Pinto
}

Departamento de Engenharia Florestal, Universidade Federal de Mato Grosso, Cuiabá - MT, Brasil.

\begin{abstract}
RESUMO A durabilidade natural da madeira é essencial na definição do seu uso. Assim, objetivou-se avaliar a deterioração ao longo de 12 meses da madeira tratada e não tratada de Corymbia citriodora, Eucalyptus camaldulensis $\mathrm{x}$ Eucalyptus grandis, e Eucalyptus camaldulensis em ensaio de campo. Os materiais de 5 anos, confeccionados em toretes de $40 \mathrm{~cm}$ de comprimento, foram tratados com Arseniato de cobre cromatado (CCA tipo C), e tiveram massa inicial determinada a $0 \%$ de umidade. Sequencialmente, foram instalados em ensaio de campo. Realizou-se três avaliações em intervalos de 4 meses, em que foram analisados o Potencial de Ataque Fúngico (PAF), Índice de Deterioração (ID) e Perda de Massa (PM). Os resultados mostraram que o PAF foi maior entre outubro e abril. Para as madeiras tratadas houve baixa PM e deterioração ao longo do tempo. Já as não tratadas sofreram maiores danos, principalmente no período entre 4 e 8 meses. O PAF apresentou pouca relação com a deterioração, visto os padrões de deterioração das madeiras; a preservação foi eficiente no aumento da durabilidade das madeiras; inicialmente a deterioração foi maior, diminuindo e se estabilizando com o tempo; os materiais genéticos não apresentaram diferenças significativas para ID e PM.
\end{abstract}

Palavras-chave: durabilidade, preservação, potencial de ataque fúngico, CCA.

\section{Deterioration of Eucalyptus and Corymbia wood in field test}

\begin{abstract}
The natural durability of wood is essential in defining its use. Thus, the objective was to evaluate the durability over time of treated and untreated wood of Corymbia citriodora, Eucalyptus camaldulensis $\mathrm{x}$ Eucalyptus grandis, and Eucalyptus camaldulensis in field test. Small logs, 5 years old, were treated with copper chromate arsenate (CCA type C), and had initial mass determined at $0 \%$ moisture. Sequentially, they were installed in field test. Three evaluations were carried out, with a fourmonth interval, analysing the Potential for Fungal Attack (PAF), Deterioration Index (ID) and Mass Loss (PM). The PAF was higher between October and April. The treated wood presented low deterioration and PM over time. The untreated ones suffered more damages, mainly in the period between 4 and 8 months. The PAF showed little relation with the deterioration, considering the deterioration of patterns of the woods; preservation was efficient in increasing wood durability; initially the deterioration was greater, decreasing and stabilizing with time; the genetic material did not present significant differences for ID and PM.
\end{abstract}

Keywords: durability, preservation, fungal attack potential, CCA.

\section{Introdução}

Uma importante característica da madeira é sua durabilidade natural, sendo determinada por meio da sua capacidade de resistir a diferentes intempéries. Romanini et al. (2014) citam como responsáveis pela deterioração da madeira, agentes xilófagos (insetos, microrganismos e brocas marinhas); e condições ambientais (exposição à luz solar, chuva e variações de temperatura), além de reações químicas diversas. Assim, conhecer a resistência da madeira frente à essas características são imprescindíveis para uma finalidade de uso adequada, bem como servem de base para melhorar a eficiência de tratamentos em madeira. 
Espécies de Eucalyptus e Corymbia comumente são utilizadas no setor de biomassa da energia (EUFRADE JUNIOR et al., 2016) e papel e celulose (NEIVA et al., 2015). Tal destinação ocorre principalmente pelo mercado já estabelecido desses setores, além disso, essas espécies comumente apresentam baixa durabilidade natural, se mantendo em condições favoráveis de uso por poucos anos, quando em contato direto com o solo (VIDAL et al., 2015; VIVIAN et al., 2014; MAGALHÃES et al., 2012; ARAÚJO et al., 2012).

A madeira tratada é empregada principalmente no setor rural, em forma de mourões de cerca; postes, para instalação de redes de energia; dormentes, para construção de estradas de ferro e em menor escala para construção civil (VIDAL et al., 2015). Mesmos autores apontam o tratamento de célula cheia (Bethell), de alta pressão, como o mais utilizado.

A madeira quando devidamente tratada, pode alcançar durabilidade entre 10 e 40 anos, variando conforme suas condições de exposição ambiental (JAMBECK et al., 2007). O aumento da vida útil da madeira, torna este material mais interessante comercialmente, visto a diminuição de trocas constantes, comuns as madeiras menos duráveis.

Nesse sentido, é imprescindível a averiguação da qualidade de tratamentos preservativos através de avaliações que determinem a durabilidade natural da madeira e a efetividade da solução preservativa. Tais resultados podem ser alcançados através de ensaios de campo, experimentos realizados sob condições reais de uso, onde as amostras, madeiras parcialmente soterradas, são expostas ao ambiente e avaliadas periodicamente (COOKSON et al., 2014). Através de ensaios de campo é possível observar quais regiões da madeira sofreram maior deterioração, e assim, relacionar a fatores ligados a degradação da madeira, como tempo de exposição, potencial de desenvolvimento de organismos xilófagos e condições ambientais do local avaliado (VIVIAN et al., 2014), possibilitando uma investigação mais clara da deterioração.
Dessa forma, na presente pesquisa objetivou-se avaliar o Potencial de Ataque Fúngico, o Índice de Deterioração e a Perda de Massa da madeira tratada com Arseniato de cobre cromatado (CCA tipo C) e não tratada de Corymbia citriodora (Hook.); Eucalyptus camaldulensis Dehn. x Eucalyptus grandis Hill e Eucalyptus camaldulensis Dehnh em ensaio de campo, ao longo de um período de 12 meses.

\section{Material e Métodos}

\section{Material}

A madeira utilizada foi proveniente de um plantio comercial de eucalipto, com espaçamento de $3 \times 3 \mathrm{~m}$ e idade de cinco anos, oriundos da Fazenda Santo André localizada no município de Chapada dos Guimarães, Mato Grosso.

Utilizou-se três diferentes materiais genéticos: Corymbia citriodora (Hook.) K.D. Hill \& L.A.S. Johnson; Eucalyptus camaldulensis Dehnh. x Eucalyptus grandis Hill (Híbrido 1277) e Eucalyptus camaldulensis Dehnh. De cada clone, foram utilizados quatro toretes de $40 \mathrm{~cm}$ de comprimento e diâmetro entre 12 e $16 \mathrm{~cm}$, da região basal e média da árvore. A parte superior das árvores foram excluídas do seccionamento, devido seu pequeno diâmetro. Para cada material genético, quatro toretes foram utilizados; dois receberam tratamento preservativo e os outros dois foram mantidos sem tratamento.

\section{Tratamento preservativo}

O tratamento preservativo dos toretes foi realizado em autoclave industrial, na Unidade de Tratamento de Madeira Santo André, localizada em Várzea Grande, Mato Grosso. A preservação foi realizada por meio do processo de célula cheia, método de Bethell, sendo o Arseniato de cobre cromatado (CCA tipo C) o preservativo utilizado. 
As etapas do processo (Figura 1) envolveram carregamento da madeira no cilindro da autoclave, aplicação de vácuo inicial a $560 \mathrm{mmHg}$ por 30 minutos, enchimento com a solução preservativa, aplicação de pressão de $12 \mathrm{kgf} / \mathrm{cm}^{2}$ por 90 minutos, retirada do produto do cilindro, vácuo final a 560 mmHg por 10 minutos e descarregamento. Após o tratamento preservativo, a madeira foi armazenada em local protegido de umidade e sem contato com solo.

\section{Condições ambientais e potencial de ataque}

\section{fúngico}

Através da estação meteorológica localizada em Várzea Grande ( $15^{\circ} 37^{\prime} 14.74^{\prime \prime}$ S; $56^{\circ} 06^{\prime} 28,88^{\prime \prime} \mathrm{O}$ ), que atende a região de Cuiabá, no Instituto Nacional de Meteorologia (INMET), levantou-se os dados de temperatura e umidade relativa média, além da precipitação diária e mensal do período entre dezembro de 2015 e dezembro de 2016 (Tabela 1).

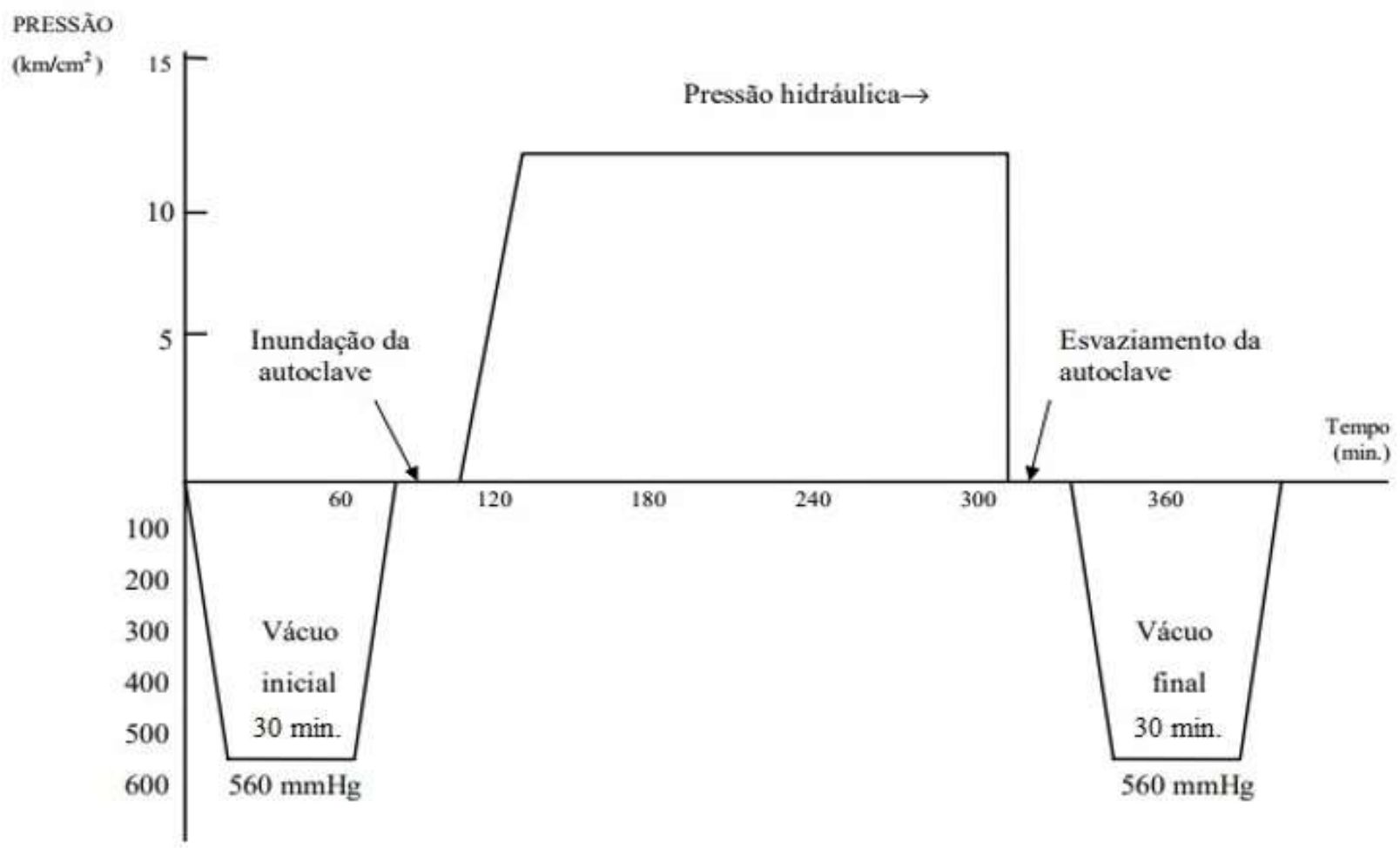

Figura 1. Representação gráfica das etapas do tratamento preservativo, baseado em Sales-Campos et al. (2003).

Figure 1. Graphical representation of the steps of the condom treatment, based on Sales-Campos et al. (2003).

Tabela 1. Valores de temperatura média $\left({ }^{\circ} \mathrm{C}\right)$, precipitação total $(\mathrm{mm})$, e umidade relativa média (\%), no período de cada avaliação.

Table 1. Values of mean temperature $\left({ }^{\circ} \mathrm{C}\right)$, total precipitation $(\mathrm{mm})$, and mean relative humidity $(\%)$, in the period of each evaluation.

\begin{tabular}{lccc}
\hline & Temperatura $\left({ }^{\circ} \mathrm{C}\right)$ & Precipitação $(\mathrm{mm})$ & Umidade Relativa $(\%)$ \\
\cline { 2 - 4 } Avaliação 1 (Dez-Abr) & 26,3 & 880,1 & 74,7 \\
Avaliação 2 (Abr-Ago) & 24,8 & 118,6 & 69,2 \\
Avaliação 3 (Ago-Dez) & 27,1 & 783,95 & 64,7 \\
\hline
\end{tabular}

Dez - Dezembro; Abr - Abril; Ago - Agosto. 
Estes dados foram coletados a fim de relacioná-los com o nível de deterioração dos toretes e o desenvolvimento de organismos xilófagos.

Para determinação do Potencial de Ataque Fúngico (PAF) utilizou-se a metodologia de Scheffer (1971), adaptada por Martins (2003), para as condições climáticas do Brasil. Os valores foram obtidos mensalmente, obtendo-se os dias com precipitação acima de $0,30 \mathrm{~mm}$ para cada mês e temperatura média mensal, conforme Equação 1.

$$
\mathrm{PAF}=\frac{(\mathrm{T}-2)(\mathrm{D}-3)}{16,7}
$$

Sendo, $\mathrm{PAF}=$ Potencial de Ataque Fúngico na madeira; $\mathrm{T}=$ temperatura média mensal; $\mathrm{D}=$ dias do mês com precipitação acima de $0,30 \mathrm{~mm}$.

\section{Ensaio de campo}

Para realização do ensaio de deterioração em campo, os toretes foram submetidos à secagem completa em estufa à 100 ${ }^{\circ} \mathrm{C}$, com circulação forçada de ar até atingirem teor de umidade igual a $0 \%$. A massa inicial seca foi determinada em balança com precisão de $0,100 \mathrm{~kg}$.

Os toretes utilizados, foram enterrados verticalmente até a sua metade $(20 \mathrm{~cm})$. Seu arranjo foi feito em um bloco sistematizado, observados em forma de croqui, conforme a Figura 2.

O ensaio de campo foi implantado em dezembro de 2015, em região de floresta secundária, próxima ao Viveiro da Faculdade de Engenharia Florestal, da UFMT campus Cuiabá, Mato Grosso.

A região do ensaio de campo possui clima com estação seca de inverno (Aw), segundo a classificação de KöppenGeiger. Durante o período do ensaio a temperatura média foi de $26,2^{\circ} \mathrm{C}$, umidade relativa média do ar de $70,20 \%$ e pluviosidade total de 1921,7 mm (Tabela 1).

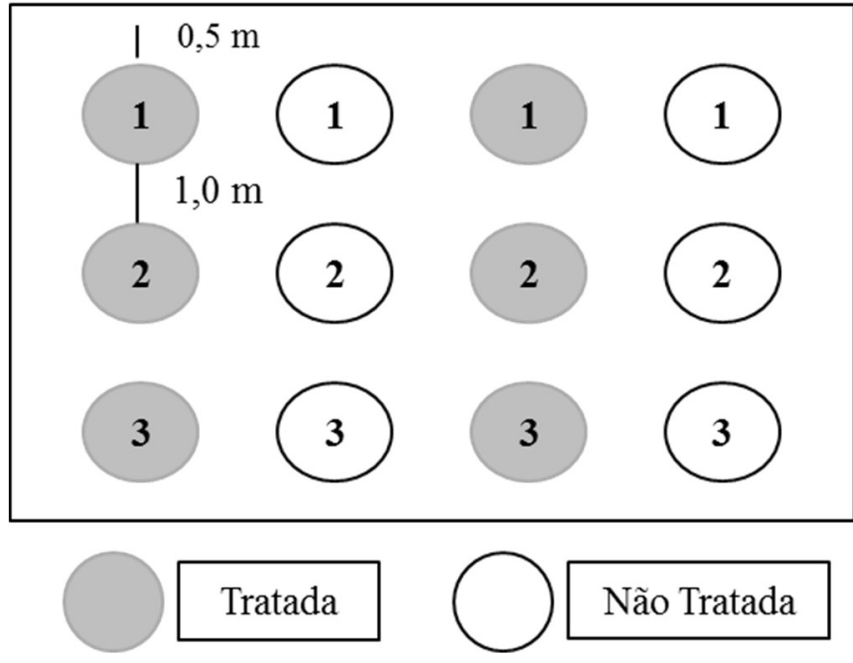

Figura 2. Distribuição das amostras dos diferentes materiais genéticos em ensaio de campo, em que 1 - Corymbia citriodora, 2 - Eucalyptus camaldulensis $\mathrm{x}$ Eucalyptus grandis, e 3 Eucalyptus camaldulensis.

Figure 2. Distribution of the samples of the different genetic materials in a field test, in which 1 - Corymbia citriodora, 2 Eucalyptus camaldulensis $\mathrm{x}$ Eucalyptus grandis, e 3 - Eucalyptus camaldulensis.

As avaliações foram realizadas com intervalos de quatro meses, em que a primeira foi realizada em abril, a segunda em agosto e a terceira em dezembro de 2016, completando-se o ciclo climático de 1 ano. Todos os toretes foram desenterrados para análise do índice de deterioração e sua perda de massa, nas três respectivas avaliações.

Assim que os toretes foram desenterrados, realizou-se a avaliação visual do índice de deterioração nas posições inferior (região com contato total com o solo), central (região de afloramento) e topo (região sem contato direto com o solo) dos toretes, conforme a classificação definida por Lepage (1986), descrita na Tabela 2.

Em seguida, os toretes foram limpos, retirando-se todo o acúmulo de solo e outros materiais que pudessem interferir na sua pesagem. Estes foram levados a estufa à $100{ }^{\circ} \mathrm{C}$ para secagem completa à $0 \%$ de umidade, sendo então calculado o valor porcentual de perda de massa, de acordo com a Equação 2. 
Tabela 2. Classificação do índice de deterioração (ID) da madeira (LEPAGE, 1986).

Table 2. Classification the of deterioration index (ID) of the wood (LEPAGE, 1986).

\begin{tabular}{ccc}
\hline Estado de sanidade & Nota & Valor \\
\hline Sadio & 0 & 100 \\
Leve à moderado & 1 & 90 \\
Moderado à intenso & 2 & 70 \\
Apodrecimento & 3 & 40 \\
intenso & 4 & 0 \\
Perda total & & \\
\hline
\end{tabular}

$$
\mathrm{PM}=\frac{\mathrm{Mi}-\mathrm{Mf}}{\mathrm{Mi}} \times 100
$$

Sendo, $\mathrm{PM}=$ perda de massa, \%; $\mathrm{Mi}=$ massa seca inicial, $\mathrm{g}$; $\mathrm{Mf}=$ massa seca final, após ensaio de campo, $\mathrm{g}$.

\section{Análise estatística}

A análise estatística foi realizada através de parcelas subdivididas no tempo, sendo os materiais genéticos e a presença, ou não, de tratamento preservativo, as variáveis relacionadas. Para a Perda de Massa realizou-se análise de variância (ANOVA) com posterior teste de Tukey em nível de 5\% de probabilidade de erro, no caso de diferença significativa entre as médias avaliadas. Para o Índice de Deterioração, utilizouse a análise de Mann Whitney para comparação das madeiras tratadas e não tratadas. Para as diferentes posições foi realizado o teste de Kruskal-Wallis, com posterior teste de Dunn a nível de 5\%, no caso de diferença significativa entre as médias avaliadas.

\section{Resultados e Discussão}

\section{Potencial de ataque fúngico}

Os valores calculados para o Potencial de Ataque Fúngico (PAF), precipitação mensal (mm) e temperatura média mensal $\left({ }^{\circ} \mathrm{C}\right)$, durante o período entre dezembro de 2015 e dezembro de 2016, conforme dados do INMET, são encontrados na Figura 3.

Na Figura 3, observa-se maior PAF no período entre dezembro de 2015 e abril de 2016, e outubro e dezembro de 2016, sendo o período entre maio e setembro de 2016 os me-

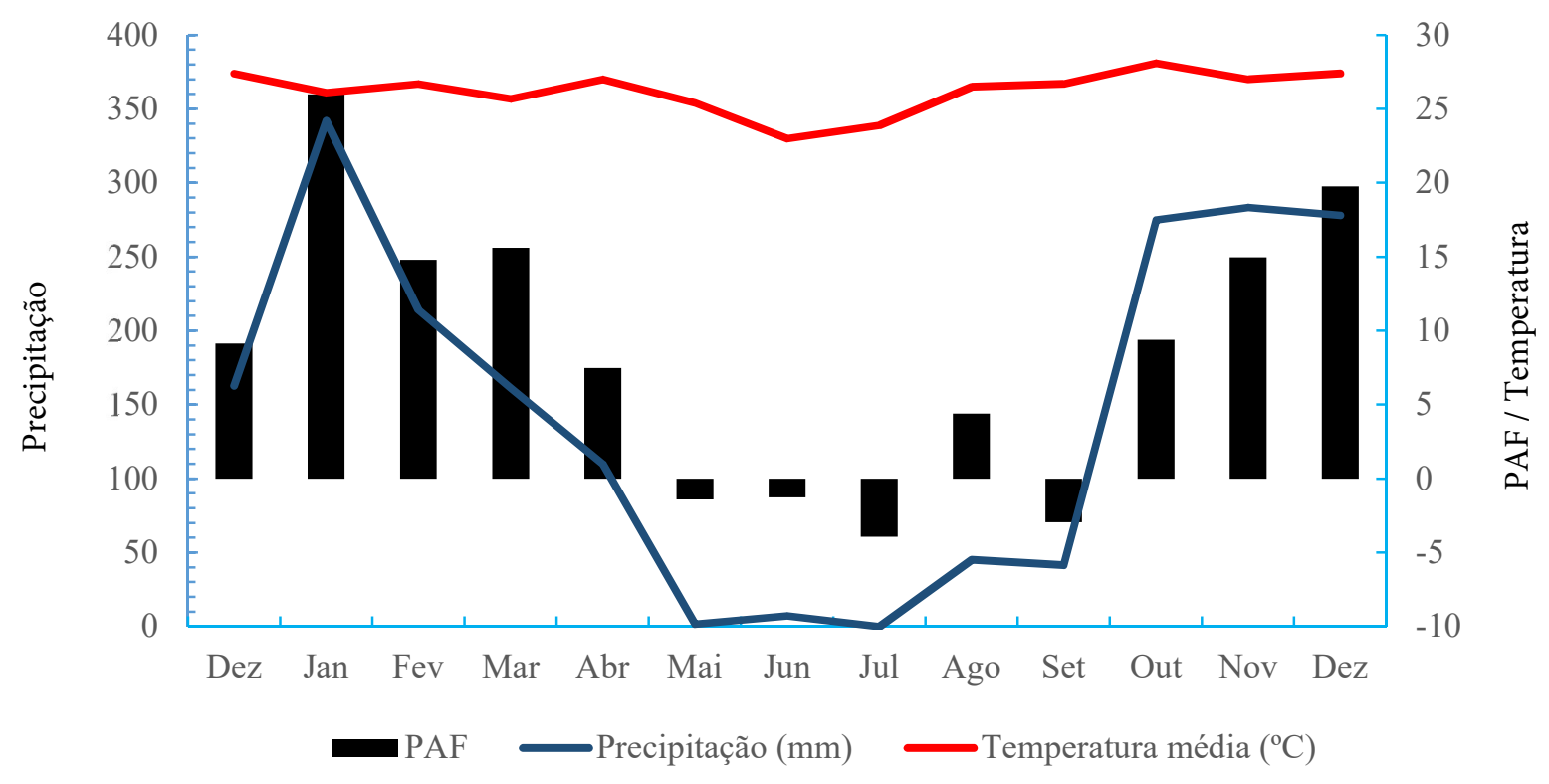

Figura 3. Potencial de ataque fúngico, precipitação $(\mathrm{mm})$ e temperatura média $\left({ }^{\circ} \mathrm{C}\right)$ no período entre dezembro de 2015 e dezembro de 2016.

Figure 3. Potential for fungal attack, precipitation $(\mathrm{mm})$ and average temperature $\left({ }^{\circ} \mathrm{C}\right)$ in the period between December 2015 and December 2016. 
nores. Ribeiro et al. (2014) em estudo sobre a durabilidade natural da madeira de Jequitibá (Cariniana micranta Ducke, Lecythidaceae) em ensaio de campo em Sinop, MT, verificaram valores similares para o PAF, para os mesmos meses do presente estudo.

O aumento encontrado para os valores de PAF está relacionado com os meses em que houveram mais dias com precipitação acima de $0,30 \mathrm{~mm}$, junto a altas temperaturas médias mensais (CARVALHO et al., 2016; CASAVECCHIA et al., 2016). O aumento do desenvolvimento de fungos, ocorre devido ao aumento da umidade e a característica de higroscopicidade da madeira, que cria na sua superfície um microclima favorável a esses microrganismos (ACCARINI et al., 2000).

A continuidade do período chuvoso também influencia nos maiores níveis de deterioração da madeira. Meyer; Brischke (2015) observaram maiores danos por fungos de podridão branca nos meses seguintes de uma estação chuvosa, visto a continuidade de condições favoráveis a esses organismos.

Os meses entre maio e setembro de 2016 apresentaram baixos valores de PAF, sendo todos negativos, exceto o mês de agosto. Este mês mostrou-se atípico, com precipitação de
$45 \mathrm{~mm}$ e mais de três dias com precipitação acima de 0,30 mm. Assim, o PAF gerado foi o maior encontrado entre os meses do mesmo período, de inverno seco, em que a frequência de chuvas raramente ultrapassa quatro ou cinco dias para todo período. Ainda, a precipitação total para o inverno comumente encontra-se entre 20 e 80 mm (SOUZA et al., 2013) e a encontrada foi de $95,3 \mathrm{~mm}$.

\section{Índice de deterioração}

Os valores do índice de deterioração (ID) das madeiras tratadas (Tabela 3) e não tratadas (Tabela 4) de Eucalyptus e Corymbia avaliadas em ensaio de campo a cada 4 meses foram classificadas conforme Lepage (1986) (Tabela 2).

Observa-se para as madeiras tratadas, que ao fim do ensaio, o ID médio de todos os materiais genético foi superior ao valor de 90 (Tabela 3), indicando baixa deterioração. Porém, para as madeiras não tratadas, notou-se ID médio inferior ao das tratadas (Tabela 4) com deterioração de moderada à intensa, exceto para o topo de Eucalyptus camaldulensis, com ID de 97,5, similar ao encontrado para as madeiras tratadas.

Tabela 3. Classificação do Índice de Deterioração (ID) da madeira tratada de Eucalyptus e Corymbia em diferentes posições dos toretes.

Table 3. Classification of the Deterioration Index (DI) of treated wood of Eucalyptus and Corymbia in different positions of the logs.

\begin{tabular}{|c|c|c|c|c|c|c|c|c|c|c|c|c|}
\hline Material Genético & \multicolumn{3}{|c|}{ Avaliação 1} & \multicolumn{3}{|c|}{ Avaliação 2} & \multicolumn{3}{|c|}{ Avaliação 3} & \multicolumn{3}{|c|}{ Médias Finais } \\
\hline Região & $\mathbf{T}$ & $\mathrm{C}$ & I & $\mathbf{T}$ & $\mathrm{C}$ & I & $\mathbf{T}$ & C & I & $\mathbf{T}$ & $\mathrm{C}$ & I \\
\hline C. citriodora & 97,5 & 100 & 97,5 & 97,5 & 100 & 95 & 97,5 & 100 & 95 & $\begin{array}{r}97,5 \\
\mathrm{Aa}\end{array}$ & $\begin{array}{l}100 \\
\mathrm{Aa}\end{array}$ & $\begin{array}{c}95,8 \\
\mathrm{Ab}\end{array}$ \\
\hline $\begin{array}{l}\text { E. camaldulensis } \\
\qquad \mathrm{X} \\
\text { E. grandis }\end{array}$ & 100 & 100 & 100 & 100 & 100 & 95 & 100 & 100 & 95 & $\begin{array}{l}100 \\
\mathrm{Aa}\end{array}$ & $\begin{array}{l}100 \\
\mathrm{Aa}\end{array}$ & $\begin{array}{r}96,7 \\
\mathrm{Ab}\end{array}$ \\
\hline E. camaldulensis & 100 & 100 & 100 & 100 & 100 & 85 & 100 & 100 & 85 & $\begin{array}{l}100 \\
\mathrm{Aa}\end{array}$ & $\begin{array}{l}100 \\
\mathrm{Aa}\end{array}$ & $\begin{array}{l}90 \\
\mathrm{Ab}\end{array}$ \\
\hline
\end{tabular}

Topo (T); Centro (C); Inferior (I). Médias seguidas na vertical, pela mesma letra maiúscula ou na horizontal, pela mesma letra minúscula, não diferem estatisticamente entre si pelo teste de Dunn a 5\% de probabilidade. 
Tabela 4. Classificação do Índice de Deterioração (ID) da madeira não tratada de Eucalyptus e Corymbia em diferentes posições dos toretes.

Table 4. Classification of the Deterioration Index (DI) of untreated wood of Eucalyptus and Corymbia in different positions of the logs.

\begin{tabular}{|c|c|c|c|c|c|c|c|c|c|c|c|c|}
\hline Material Genético & \multicolumn{3}{|c|}{ Avaliação 1} & \multicolumn{3}{|c|}{ Avaliação 2} & \multicolumn{3}{|c|}{ Avaliação 3} & \multicolumn{3}{|c|}{ Médias Finais } \\
\hline Região & $T$ & $\mathrm{C}$ & I & $T$ & $\mathrm{C}$ & I & $\mathrm{T}$ & $\mathrm{C}$ & I & $\mathrm{T}$ & $\mathrm{C}$ & I \\
\hline C. citriodora & 92,5 & 75 & 75 & 82,5 & 55 & 40 & 82,5 & 47,5 & 40 & $\begin{array}{c}85,3 \\
\mathrm{Aa}\end{array}$ & $\begin{array}{c}59,2 \\
\mathrm{Ab}\end{array}$ & $\begin{array}{c}51,7 \\
\mathrm{Ab}\end{array}$ \\
\hline $\begin{array}{c}\text { E. camaldulensis } \\
\mathrm{x} \\
\text { E. grandis }\end{array}$ & 87,5 & 90 & 80 & 87,5 & 55 & 40 & 87,5 & 55 & 20 & $\begin{array}{c}87,5 \\
\mathrm{Aa}\end{array}$ & $\begin{array}{c}66,7 \\
\mathrm{Ab}\end{array}$ & $\begin{array}{c}46,7 \\
\mathrm{Ab}\end{array}$ \\
\hline E. camaldulensis & 97,5 & 90 & 90 & 97,5 & 75 & 40 & 97,5 & 70 & 40 & $\begin{array}{r}97,5 \\
\mathrm{Aa}\end{array}$ & $\begin{array}{c}78,3 \\
\mathrm{Ab}\end{array}$ & $\begin{array}{c}56,7 \\
\mathrm{Ab}\end{array}$ \\
\hline
\end{tabular}

Topo (T); Centro (C); Inferior (I). Médias seguidas na vertical, pela mesma letra maiúscula ou na horizontal, pela mesma letra minúscula, não diferem estatisticamente entre si pelo teste de Dunn a 5\% de probabilidade.

Ao avaliar o índice de deterioração nas diferentes posições da madeira, em ensaio de campo, é possível observar que a deterioração está ligada diretamente ao seu maior contato com o solo, conforme Tabelas 3 e 4 . Todas as posições entre as madeiras tratadas e não tratadas, apresentaram diferenças significativas, sendo o ID para as madeiras tratadas superior as não tratadas nas diferentes posições, comprovando a efetividade da preservação ao longo do torete.

Entre as madeiras tratadas, observou-se maior ID na região topo e central, sendo a inferior a mais deteriorada. Já entre as não tratadas, o maior ID encontrou-se na região topo, sendo a central e inferior as mais deterioradas. Resultado semelhante ao de Araújo et al. (2012), ao avaliarem a madeira de Corymbia citriodora tratada com CCA e não tratada, por 47 meses, onde a posição inferior dos toretes, foi a mais deteriorada em ensaio de campo, visto o solo ser um dos meios em que há maior desenvolvimento de organismos xilófagos, devido ser composto em parte, por matéria orgânica, além de reter muita umidade (MELO et al., 2010; CARVALHO et al., 2016).

A efetividade da preservação é comprovada através da comparação entre as madeiras tratadas e não tratadas, descritas nas Tabelas 3 e 4, em que os valores do ID para as tratadas são superiores em relação as não tratadas. Ou seja, apresentaram maior resistência ao ataque dos organismos xilófagos. Tendência comum a outros trabalhos, onde espécies de $E$ ucalyptus após tratamento preservativo com CCA mostraram alta resistência em ensaio de campo (VIVIAN et al., 2014; MAGALHÃES et al., 2012; ARAÚJO et al., 2012).

Contudo, mesmo após tratamento preservativo, as madeiras apresentaram alguma deterioração, com maior ocorrência na posição inferior dos toretes, e região interna do cerne, conforme observado em Eucalyptus camaldulensis e Tabela 3. A deterioração da região interna do cerne, se dá ao fato de que é comum a diversas espécies, uma maior concentração de extrativos na região externa do cerne, e assim, uma maior durabilidade (PANSHIN e DE ZEEUW, 1980; OLIVEIRA et al., 1986). Mesmos autores afirmam, que com o passar do tempo e à medida que a árvore envelhece, os extrativos da região interna perdem parte da sua toxicidade.

Ainda, a deterioração do cerne após tratamento preservativo, pode ser explicada por Lopes et al. (2017), que aponta a dificuldade na penetração e retenção de soluções preservativas na região do cerne de espécies do gênero Eucalyptus e Corymbia. O cerne por ser obstruído por tilos, gomas, extrativos e outras substâncias, é impermeável, não permitindo 
preservação. Porém, gera maior durabilidade natural à madeira, visto que tais substâncias são tóxicas a organismos xilófagos.

Ao observar as diferentes avaliações, nota-se que o período correspondente a primeira avaliação, mesmo coincidindo a um intervalo de altos valores de PAF, conforme Figura 3, não apresentou alta deterioração. Já no período em que o PAF se mostrou baixo, referente a avaliação 2, a deterioração é elevada, conforme Figura 4.

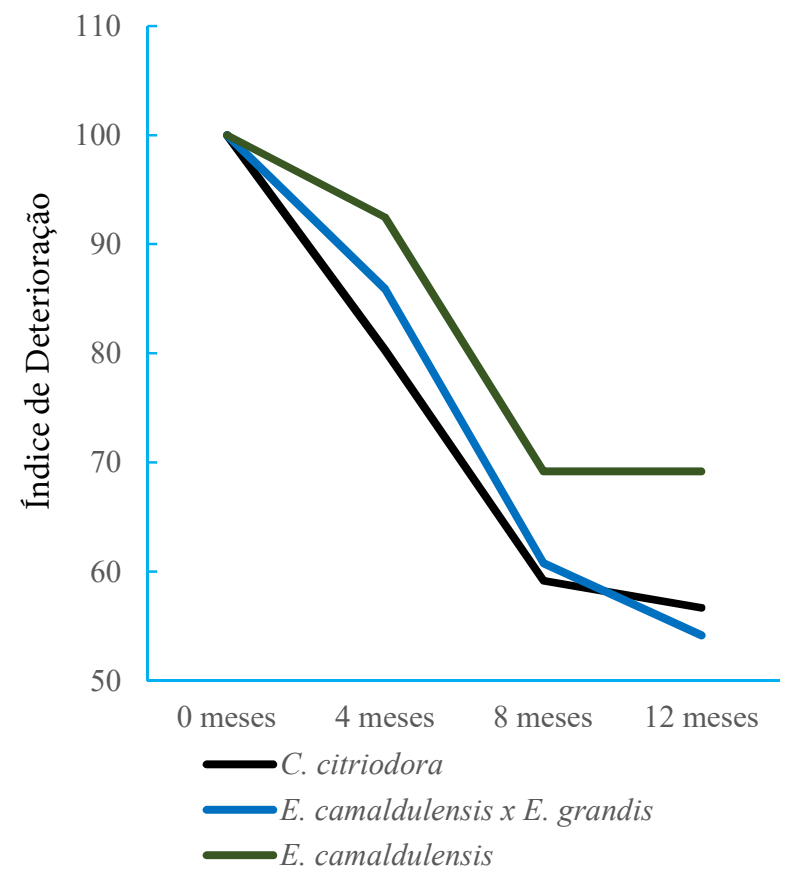

Figura 4. Variação do Índice de Deterioração (ID) das madeiras não tratadas ao longo de 12 meses, através de 3 avaliações, sendo, 4 meses (avaliação 1), 8 meses (avaliação 2), 12 meses (avaliação 3).

Figure 4. Variation of the Deterioration Index (DI) of untreated wood over 12 months through 3 evaluations, being, 4 months (evaluation 1), 8 months (evaluation 2), 12 months (evaluation 3).

No caso da terceira avaliação o PAF volta a valores altos, porém, a deterioração mantém-se baixa. Para compreender tal resultado, é necessário atentar-se ao tipo de organismo xilófago que está deteriorando a madeira. No presente trabalho, mesmo não havendo o levantamento dos organismos xilófagos, é notável a baixa ocorrência de fungos, visto os padrões de deterioração das madeiras (Figura 5 e 6) e a baixa relação observada entre PAF e ID.

De modo geral, observa-se que nas avaliações 1 e 2 , período entre 0 e 8 meses (dezembro/2015 à agosto/2016), há um maior declínio dos valores de ID para as madeiras não tratadas, principalmente no período de abril à agosto, referente a avaliação 2 (Figura 4). Contudo, ao observar a avaliação 3, período entre 8 e 12 meses (agosto/2016 à dezembro/2016), a diminuição dos valores de ID são mínimas. No caso das madeiras tratadas a deterioração também é maior nesse mesmo período, no entanto, numa proporção menor que para as madeiras não tratadas.

O aumento da deterioração ocorrido no período entre as avaliações 1 e 2, principalmente para as madeiras não tratadas, pode ser explicado pela facilidade em que componentes de menor peso molecular, como açúcares simples, amido e proteínas podem ser degradados por organismos xilófagos (EATON e HALE, 1993). Após a degradação desses componentes, a deterioração passa a ser mais lenta, justificando os resultados observados no período entre as avaliações 2 e 3, principalmente para Eucalyptus camaldulensis, em que a deterioração nesse período é mínima. Tais resultados apontam que a deterioração a curto prazo, sofre uma maior influência da degradação dos componentes de baixo peso molar, do que, valores de PAF.

\section{Perda de massa por avaliação}

A perda de massa (PM) verificada para as madeiras de $E$ ucalyptus e Corymbia em ensaio de campo, são encontradas na Tabela 5, onde as avaliações foram realizadas com intervalos de 4 meses. Os valores descritos são específicos a cada avaliação.

De acordo com os resultados apresentados na Tabela 5, não houve efeito significativo do material genético na perda 
de massa. No entanto, verificou-se significância do tratamento preservativo, onde as madeiras não tratadas apresentaram maior perda de massa em todas as avaliações.

Entre as avaliações não houve diferenças significativas de perda de massa, ou seja, a perda de massa foi constante ao longo do tempo, mesmo com períodos de mudanças climáticas, principalmente em relação a pluviosidade, como observado na Figura 3.

Outro fator a ser analisado é a estrutura florestal, que apresenta no seu microclima, uma tendência a equilibrar valores de umidade e temperatura, diminuindo o efeito individual da variação climática na deterioração. Essa estabilidade ambiental decorrente de ambientes florestais justifica a não correlação da perda de massa em função das condições climáticas das avaliações individualmente, conforme Tabela 5.

Vivian et al. (2014) afirmam que temperaturas entre $5{ }^{\circ} \mathrm{C}$ e $35{ }^{\circ} \mathrm{C}$ são as mais propícias para o desenvolvimento da maior parte dos microrganismos, sendo essa faixa ocorrida durante todo o período do ensaio. Araújo et al. (2012) apontam que condições tropicais, de alta umidade e elevada temperatura, como as do presente estudo, favorecem uma maior perda de massa de madeiras não tratadas (Tabela 6). Visto esse habitat possuir uma menor variação ambiental, gerando assim, uma condição favorável e estável a grande parte dos organismos xilófagos (TREVISAN et al., 2008; VIVIAN et al., 2014).

Tabela 5. Perda de massa média (\%) das madeiras tratadas (T) e não tratadas (NT), em cada avaliação.

Table 5. Average mass loss (\%) of the treated (T) and untreated (NT) woods, in each evaluation.

\begin{tabular}{ccc|c|c|c|c}
\hline \multirow{2}{*}{ Material Genético } & \multicolumn{2}{c|}{ Avaliação 1 } & \multicolumn{2}{c}{ Avaliação 2 } & \multicolumn{2}{c}{ Avaliação 3 } \\
\cline { 2 - 7 } & $\mathbf{T ~ ( \% )}$ & NT (\%) & T (\%) & NT (\%) & T (\%) & NT (\%) \\
\hline C. citriodora & $1,76 \mathrm{Aa}$ & $7,78 \mathrm{Ab}$ & $0,14 \mathrm{Aa}$ & $5,11 \mathrm{Ab}$ & $1,04 \mathrm{Aa}$ & $4,60 \mathrm{Ab}$ \\
E. grandis $x$ E. camaldulensis & $2,53 \mathrm{Aa}$ & $6,67 \mathrm{Ab}$ & $0,99 \mathrm{Aa}$ & $14,22 \mathrm{Ab}$ & $0,29 \mathrm{Aa}$ & $4,59 \mathrm{Ab}$ \\
E. camaldulensis & $1,58 \mathrm{Aa}$ & $5,31 \mathrm{Ab}$ & $3,42 \mathrm{Aa}$ & $6,72 \mathrm{Ab}$ & $0,14 \mathrm{Aa}$ & $2,81 \mathrm{Ab}$ \\
\hline
\end{tabular}

Médias seguidas na vertical, pela mesma letra maiúscula ou na horizontal, pela mesma letra minúscula, não diferem estatisticamente entre si pelo teste de Tukey a 5\% de probabilidade de erro.
Dessa forma, é altamente importante a caracterização das condições ambientais de cada região, associadas a durabilidade da madeira e ao comportamento e desenvolvimento dos diferentes organismos xilófagos. A partir do momento em que for levantado dados consideráveis dessas relações, será possível definir quais espécies possuem maior resistência as intempéries de cada região, bióticas, ou abióticas, potencializando seu uso.

\section{Perda de massa acumulada}

A perda de massa acumulada ao decorrer do tempo representada na Tabela 6, traz os valores obtidos para as madeiras tratadas e não tratadas avaliadas no ensaio de campo.

Não houve efeito significativo do material genético na perda de massa. Além disso, a perda de massa em função do tempo ( 4,8 e 12 meses) para as madeiras tratadas mostrou-se baixa e similar ao longo do experimento, conforme Tabela 6 e Figura 5. Vivian et al. (2014) ao analisarem a PM de diferentes clones de eucalipto, submetidos a preservação em autoclave com CCA, ao longo de 300 dias, não obtiveram diferenças entre os materiais genéticos e o tempo de exposição em ensaio de campo para as madeiras tratadas, resultados similares ao presente estudo.

Devido a deterioração ocorrer com maior intensidade na posição inferior dos toretes, as Figuras 5 e 6 apresentam 
Tabela 6. Perda de massa média (\%) acumulada por avaliação das madeiras tratadas e não tratadas.

Table 6. Average mass loss (\%) accumulated by evaluation of treated and untreated woods.

\begin{tabular}{|c|c|c|c|c|c|c|}
\hline \multirow{2}{*}{ Material Genético } & \multicolumn{2}{|c|}{ Avaliação 1} & \multicolumn{2}{|c|}{ Avaliação 2} & \multicolumn{2}{|c|}{ Avaliação 3} \\
\hline & $\mathrm{T}(\%)$ & NT (\%) & $\mathrm{T}(\%)$ & NT (\%) & $\mathrm{T}(\%)$ & NT (\%) \\
\hline $\begin{array}{l}\text { C. citriodora } \\
\text { E. grandis }\end{array}$ & $1,76 \mathrm{Aa}$ & $7,78 \mathrm{Ab}$ & $1,90 \mathrm{Aa}$ & $12,48 \mathrm{Ac}$ & $2,91 \mathrm{Aa}$ & $16,50 \mathrm{Ac}$ \\
\hline $\begin{array}{c}\mathrm{x} \\
\text { E. camaldulensis }\end{array}$ & $2,53 \mathrm{Aa}$ & $6,67 \mathrm{Ab}$ & $3,52 \mathrm{Aa}$ & $19,97 \mathrm{Ac}$ & $3,81 \mathrm{Aa}$ & $23,56 \mathrm{Ac}$ \\
\hline E. camaldulensis & $1,58 \mathrm{Aa}$ & $5,31 \mathrm{Ab}$ & $5,01 \mathrm{Aa}$ & $11,68 \mathrm{Ac}$ & $5,15 \mathrm{Aa}$ & $14,16 \mathrm{Ac}$ \\
\hline
\end{tabular}

Médias seguidas na vertical, pela mesma letra maiúscula ou na horizontal, pela mesma letra minúscula, não diferem estatisticamente entre si pelo teste de Tukey a 5\% de probabilidade de erro.
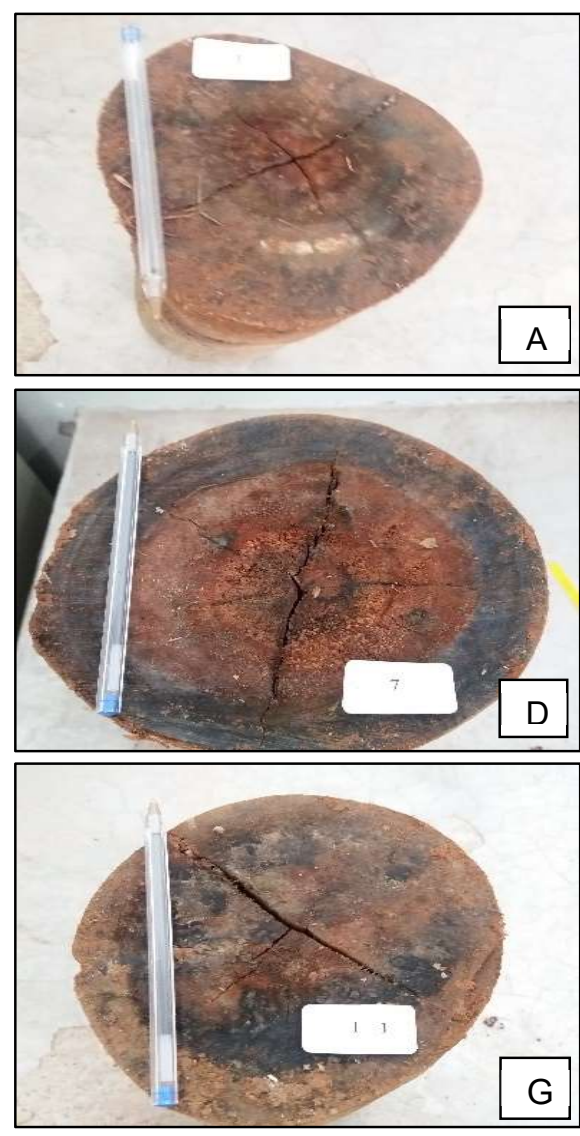
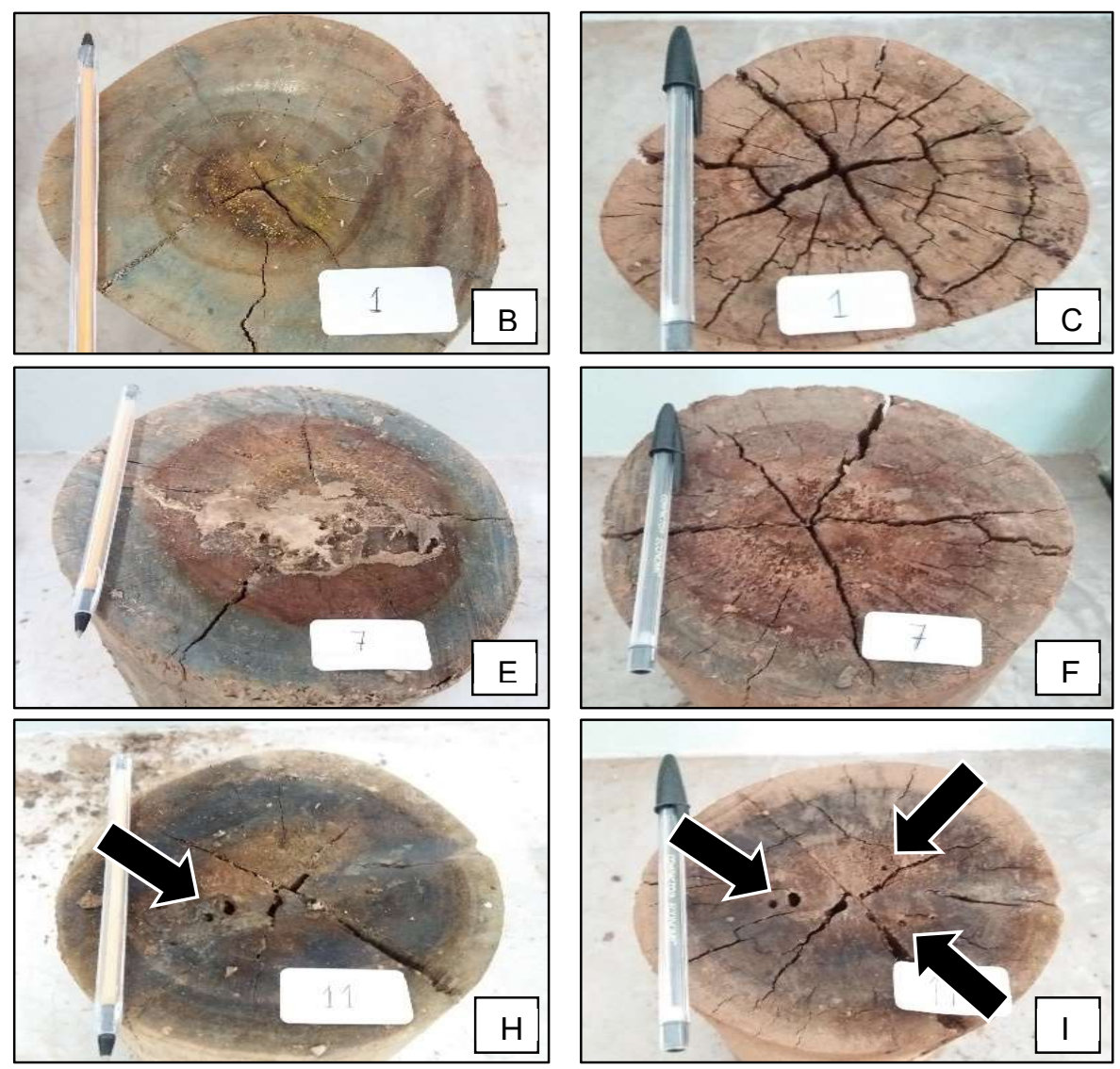

Figura 5. Avaliação visual da região inferior das madeiras tratadas ao longo do tempo. Sendo, 4 meses (A, D, G), 8 meses (B, E, H) e 12 meses (C, F, I). Onde Corymbia citriodora (A, B, C), Eucalyptus camaldulensis x Eucalyptus grandis (D, E, F), e Eucalyptus camaldulensis $(\mathrm{G}, \mathrm{H}, \mathrm{I})$. Setas indicando deterioração da madeira por cupins.

Figure 5. Visual evaluation of the lower region of treated wood over time. Being 4 months (A, D, G), 8 months (B, E, H) and 12 months (C, F, I). Where Corymbia citriodora (A, B, C), Eucalyptus camaldulensis x Eucalyptus grandis (D, E, F), and Eucalyptus camaldulensis $(\mathrm{G}, \mathrm{H}, \mathrm{I})$. Arrows indicating deterioration of wood by termites.

apenas essa porção, e sua deterioração, ao longo do tempo.

Ao analisar a deterioração das madeiras tratadas (Figura 5), observa-se que mesmo com o aumento do tempo de exposição, houve pouca variação da deterioração, visto o aumento de durabilidade após tratamento preservativo, reiterando os valores encontrados na Tabela 6. Exceto para Eucalyptus camaldulensis, conforme setas indicativas da Figura 5, que apresentam a deterioração por cupins na região do cerne, a partir da avaliação 2, aos 8 meses de exposição em ensaio de campo.

Como a região do alburno preservado possui alta durabilidade e o cerne geralmente possuir razoável resistência 
natural à organismos xilófagos, devido as substâncias fenólicas encontradas no seu lenho (PEREIRA, et al., 2013) grandes aumentos de perda de massa não são esperados em períodos curtos de tempo. No entanto, ao comparar-se com as madeiras não tratadas, a perda de massa diverge, conforme Tabela 6.

Para as madeiras não tratadas, observou-se na primeira avaliação valores menores de perda de massa, sendo a segunda e terceira avaliações, maiores, e iguais estatisticamente entre si. Tal comportamento é visível na Figura 6, onde a região inferior dos toretes sem tratamento preservativo é observada ao longo do tempo. A deterioração avaliada mostra-se inferior na primeira avaliação e maior na segunda e terceira avaliações, sendo estas, similares.
A perda de massa se mostra crescente e sempre maior que a encontrada na avaliação anterior (Tabela 6). Segundo Moreschi (1996), a alta umidade e a temperatura, como as encontradas neste estudo, são favoráveis ao desenvolvimento de cupins subterrâneos, o que justifica a alta incidência desse organismo xilófago (Figuras 5 e 6).

A maior ocorrência de cupins corresponde com o acúmulo da perda de massa das madeiras não tratadas ao decorrer do tempo. Hunt; Garrat (1953) explicam que os cupins inicialmente têm preferência pela região mais macia da madeira, no sentido das fibras. Contudo, quando esta se torna escassa, a porção mais dura também é deteriorada, sendo natural que a perda de massa seja constantemente acrescida,
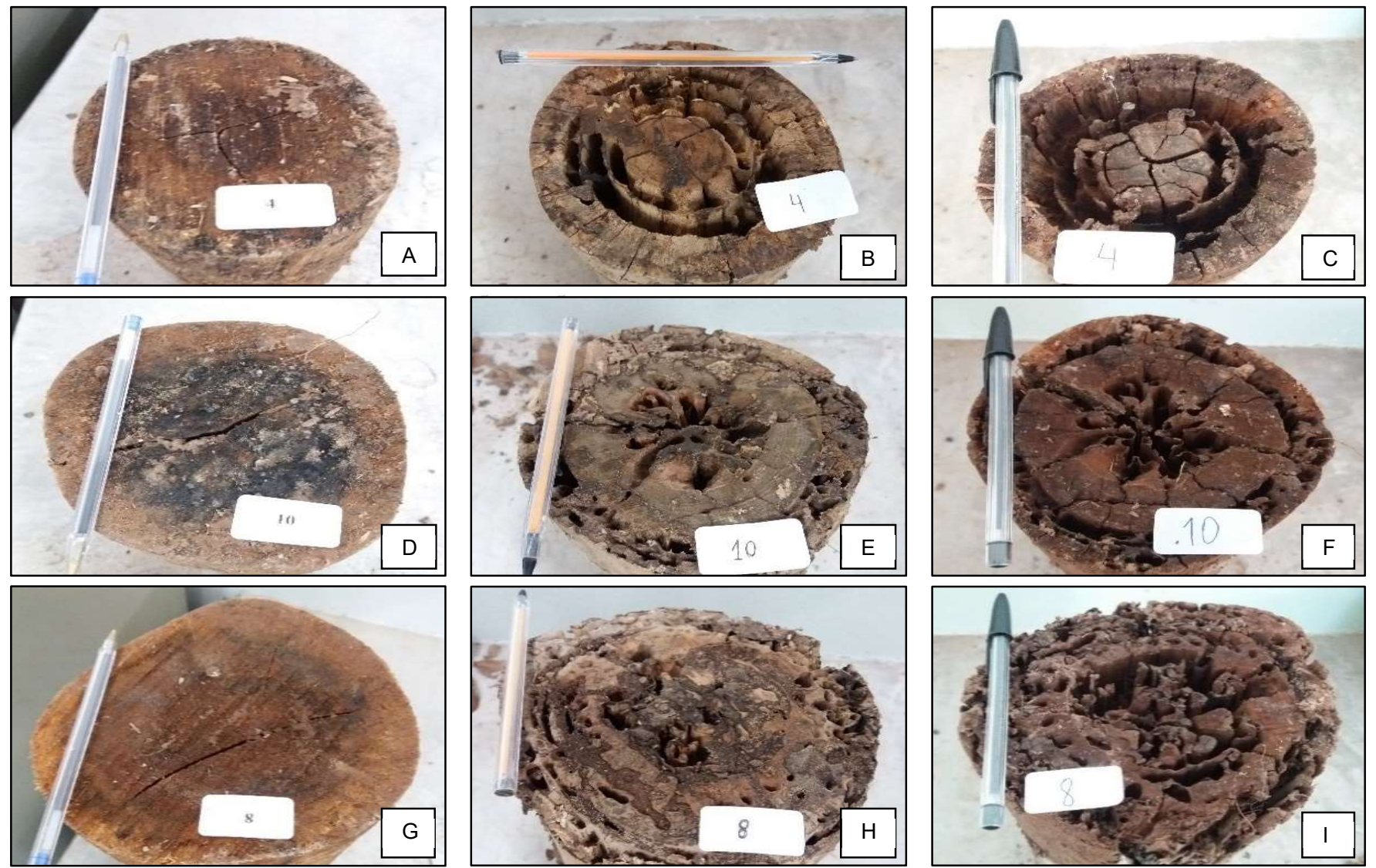

Figura 6. Avaliação visual da região inferior das madeiras não tratadas ao longo do tempo. Sendo, 4 meses (A, D, G), 8 meses (B, E, H) e 12 meses (C, F, I). Onde Corymbia citriodora (A, B, C), Eucalyptus camaldulensis x Eucalyptus grandis (D, E, F), e Eucalyptus camaldulensis (G, H, I).

Figure 6. Visual evaluation of the lower region of untreated wood over time. Being 4 months (A, D, G), 8 months (B, E, H) and 12 months (C, F, I). Where Corymbia citriodora (A, B, C), Eucalyptus camaldulensis $\mathrm{x}$ Eucalyptus grandis (D, E, F), and Eucalyptus camaldulensis $(\mathrm{G}, \mathrm{H}, \mathrm{I})$ 
principalmente para as madeiras não tratadas, como observado na Tabela 6 e Figura 6.

Resultados semelhantes de perda de massa foram verificados por diversos autores que avaliaram diferentes madeiras tratadas e não tratadas em ensaio de campo (MELO et al., 2010; ARAÚJO et al., 2012; CORASSA et al., 2013; MATTOS et al., 2013; VIVIAN et al., 2014).

\section{Conclusões}

O Potencial de Ataque Fúngico não apresentou resposta direta quanto a deterioração e a perda de massa, contudo, apresenta potencial como indicador da ocorrência de fungos xilófagos.

O tratamento preservativo com CCA mostrou-se eficiente no aumento da durabilidade das madeiras de Corymbia citriodora, Eucalyptus camaldulensis x Eucalyptus grandis e Eucalyptus camaldulensis nas diferentes posições do torete.

Não houve efeito significativo do material genético sobre o índice de deterioração e a perda de massa, tanto para as madeiras tratadas, quanto para as não tratadas.

A deterioração foi maior no início da exposição, de acordo com a degradação dos compostos de menor peso molar, diminuindo e estabilizando-se com o passar do tempo.

\section{Agradecimentos}

Os autores agradecem ao Conselho Nacional de Desenvolvimento Científico e Tecnológico - CNPq e à empresa Eucalipto Tratado Santo André pelo apoio prestado ao desenvolvimento deste trabalho.

\section{Referências}

ACCARINI, J. H.; MAZOCATO, M. A.; COSTA, O. G. P.; LUENGO, R. F. A. Pontos de estrangulamento: os obstáculos internos e externos representam vicissitudes a serem vencidas pela olericultura brasileira. Agroanalysis, Rio de Janeiro - RJ, v. 20, n. 2, p. 32-36, 2000.

ARAÚJO, H. J. B. D.; MAGALHÃES, W. L. E.; OLIVEIRA, L. C. D. Durabilidade de madeira de eucalipto citriodora (Corymbia citriodora (Hook.) K.D. Hill \& L.A.S. Johnson) tratada com CCA em ambiente amazônico. Acta Amazonica, Manaus - AM, v. 42, n. 1, p. 49-58, 2012.

CARVALHO, D. E. et al. Natural durability of Eucalyptus dunnii Maiden, Eucalyptus robusta Sm., Eucalyptus tereticornis Sm. and Hovenia dulcis Thunb. Wood in field and forest environment. Revista Árvore, Viçosa - MG, v.40, n.2, p.363$370,2016$.

CASAVECCHIA, B. H. et al. Potential fungal attack for wood in Mato Grosso state, Brazil. Nativa, Sinop - MT, v. 4, n. 3, p. 156-161, 2016.

COOKSON, L. J.; PAGE, D.; SINGH, T. Accelerated aboveground decay testing in Australia and New Zealand. International Biodeterioration \& Biodegradation, v. 86, p. 210-217, 2014.

CORASSA, J. N. Durabilidade natural da madeira de quatro espécies florestais em ensaios de deterioração em campo. Ciência da Madeira, Pelotas, v. 04, n. 01, p. 108-117, 2013.

EATON, R. A.; HALE, M. D. C. Wood: decay, pests and protection. London: Chapman \& Hall, 1993. p. 546.

EUFRADE JUNIOR, H. J.; OGURI, G.; MELO, R. X.; BALLARIN, A. W.; GUERRA, S. P. S. Storage of whole-tree chips from high-density energy plantations of Eucalyptus in Brazil. Biomass and Bioenergy, v. 93, p. 279-283, 2013.

HUNT, G. M.; GARRATT, G. A. Wood preservation. 3. Ed. New York: McGraw-Hill, 1953. 417 p.

JAMBECK, J.; WEITZ, K.; SOLO-GABRIELE, H.; TOWNSEND, T.; THORNELOE, S. CCA-Treated wood disposed in landfills and life-cycle trade-offs with waste-to-energy and MSW landfill disposal. Waste Management, 27 (8), S21-S28, 2007.

LEPAGE, E. S. Manual de preservação de madeiras. São Paulo: IPT. 2. Ed., 1986. 708 p.

LOPES, D. J. V.; PAES, J. B.; JANKOWSKY, I. P.; SEGUNDINHO, P. G. A.; VIDAURRE, G. B. Influences of Diameter and Wood Moisture on Quality of the Preservative Treatment. Floresta e Ambiente, v. 24, 2017. 
MAGAlHÃES, W. L. E.; MATTOS, B. D.; MISSIO, A. L. Field testing of CCA-treated Brazilian spotted gum. International Biodeterioration \& Biodegradation, n. 74, p. 124-128, 2012.

MARTINS, V. A.; ALVES, M. V. S.; SILVA, J. de F.; REBELLO, E. R. G.; PINHO, G. S. C. de. Umidade de equilíbrio e risco de apodrecimento da madeira em condições de serviço no Brasil. Brasil Florestal, Brasília - DF, n. 7 6, p. 29-34, 2003.

MATTOS, B. D.; CADEMARTORI, P. H. G.; STANGERLIN, D. M.; BELTRAME, R. Durabilidade a campo da madeira de três espécies de Eucalyptus tratadas por imersão simples. Revista Brasileira de Ciências Agrárias. Recife - PE, v. 8, n. 4, p. 648-655, 2013.

MELO, R. R.; STANGERLIN, D. M.; SANTINI, E. J.; HASELEIN, C. R.; GATTO, D.A.; SUSIN, F. Durabilidade da madeira de três espécies florestais em ensaios de campo. Ciência Florestal, Santa Maria - RS, v. 20, n. 2, p. 357-365, 2010.

MEYER, L.; BRISCHKE, C. Fungal decay at different moisture levels of selected European-grown wood species. International Biodeterioration \& Biodegradation, Barking, v. 103, p. 23-29, 2015

MORESCHI, J. C. Biodeterioração e Preservação da Madeira. Revista da Madeira. Curitiba - PR, n. 43, p. 46-52, 1996.

NEIVA, Duarte et al. Chemical composition and kraft pulping potential of 12 eucalypt species. Industrial Crops and Products, n. 66, p. 89-95, 2015.

OLIVEIRA, A. M. F., LELIS, A. T., LEPAGE, E. S., CARBALLERA LOPEZ, G. A., OLIVEIRA, L. C. S., CANEDO, M. D., MILANO, S. Agentes destruidores da madeira. In: LEPAGE, E. S. (Coord.) Manual de preservação de madeiras. São Paulo: IPT, 1986. v. 1 p. 99-279.

PANSHIN, A. J; DE ZEEUW, C. Textbook of wood technology. 4 ed. New York: Mack Graw-Hill, 1980, 722p.

PEREIRA, B. L. C.; OLIVEIRA, A. C.; CARVALHO, A. M. M. L.; CARNEIRO, A. C. O.; VITAL, B. R.; SANTOS, L. C. Correlações entre a relação Cerne/Alburno da madeira de eucalipto, rendimento e propriedades do carvão vegeta. Scientia Forestalis, Piracicaba - SP, v. 41, n. 98, p. 217-225, 2013.

RIBEIRO, M. A.; STANGERLIN, D. M.; SOUZA, A. P.; CARDOSO, G. V.; CALERIGARI, L.; GATTO, D. A. Durabilidade natural da madeira de jequitibá em ensaios de deterioração em campo aberto e floresta durante as estações de seca e chuva. Comunicata Scientiae, Bom Jesus - PI, v. 5, n. 4, p. 402-411, 2014.

ROMANINI, A.; STANGERLIN, D. M.; PARIZ, E.; SOUZA, A. P.; GATTO, D. A.; CALEGARI, L. Durabilidade natural da madeira de quatro espécies amazônicas em ensaios de deterioração de campo. Nativa, Sinop - MT, v. 2, n. 1, p. 13-21, 2014.

SALES-CAMPOS, C.; VIANEZ, B. F.; MENDONÇA, M. S. Estudo da variabilidade da retenção do preservante CCA tipo A na madeira de Brosimum rubescens taub. Moraceae - (PauRainha) uma espécie madeireira da região amazônica. Revista Árvore, Viçosa-MG, v. 27, n. 6, p. 845-853, 2003.

SCHEFFER, T. C. A climate index for estimating potential for decay in wood structures above ground. Forest Products Journal, Madison - WI, v. 21, n. 10, p. 25-31, 1971.

SOUZA, A.P.; MOTA, L.L.; ZAMADEI, T.; MARTIM, C.C.; ALMEIDA, F.T., PAULINO, J. Classificação climática e balanço hídrico climatológico no estado de Mato Grosso. Nativa, Sinop - MT, v. 1, n. 1, p. 34-43, 2013.

TREVISAN, H.; MARQUES, F. M. T.; CARVALHO, A. G. Degradação natural de toras de cinco espécies florestais em dois ambientes. Floresta, Curitiba - PR, v. 38, n. 1, p. 33-41, 2008 .

VIDAL, J. M.; EVANGELISTA, W. V.; SILVA, J. C.; JANKOWSKY, I. P. Preservação de madeiras no Brasil: histórico, cenário atual e tendências. Ciência Florestal, Santa Maria RS, v. 25, n. 1, p. 257-271, 2015.

VIVIAN, M. A.; SANTINI, E. J.; MODES, K. S.; CARVALHO, D. E.; MORAIS, W. W. C. Resistência biológica da madeira tratada de duas espécies de Eucalyptus em ensaio de campo. Pesquisa Florestal Brasileira, Colombo - PR, v. 34, n. 80, p. $425-433,2014$. 